\title{
ENTRE DEMOCRACIA Y DICTADURA: INCLUSIÓN EN LA EDUCACIÓN SUPERIOR EN PAÍSES DE LATINOAMÉRICA, EUROPA ORIENTAL Y EUROPA OCCIDENTAL (1950-2000)
}

\author{
Enrique Fernández Darraz ${ }^{1}$, Robert Reisz ${ }^{2}$, \\ Manfred Stock ${ }^{3}$
}

\begin{abstract}
RESUMEN
El artículo analiza la relación que existe entre las condiciones sociopolíticas y la inclusión en la educación superior. Para ello contrasta dicha situación en Europa Occidental, con lo sucedido en países de Europa del Este que vivieron bajo un socialismo de Estado y en países latinoamericanos que fueron gobernados por dictaduras militares. El periodo analizado comprende desde 1950 hasta el año 2000. La principal hipótesis es que las condiciones sociopolíticas tienen incidencia en los procesos de expansión de la educación superior, de modo que esta no constituye un proceso de expansión continua como sostienen las teorías neoinstitucionales. Finalmente, para explicar la relación entre las condiciones sociopolíticas y la educación superior se recurre a las teorías sociológicas de la diferenciación funcional y de sistemas.
\end{abstract}

Palabras clave: Dictadura, democracia, educación superior, expansión.

\section{BETWEEN DEMOCRACY AND DICTATORSHIP: HIGHER EDUCATION INCLUSION IN COUNTRIES OF LATIN AMERICA, EASTERN AND WESTERN EUROPE (1950-2000)}

\begin{abstract}
This article analyses the relationship between socio-political conditions and inclusion in higher education. In doing so, the article contrasts the situation in Western Europe and the situation of Eastern European countries that lived under state socialism and Latin American countries ruled by military dictatorships. The study covers the period from 1950 to 2000. The main hypothesis is that socio-political conditions influence expansion processes in higher education and therefore it is not a process of constant expansion as new institutionalism theories have established. Finally, it uses sociological theories of functional differentiation and systems to explain the relationship between socio-political conditions and higher education.
\end{abstract}

Keywords: Dictatorship, democracy, higher education, expansion

1 Enrique Fernández Darraz, Universidad de Tarapacá, Santiago, Chile. Contacto: efernandezdarraz@gmail.com

2 Universidad de Timisoara, Timisoara, Rumania.

3 Universidad de Halle-Wittenberg, Halle-Wittenberg, Alemania. 


\section{ENTRE DEMOCRACIA Y DICTADURA: \\ INCLUSIÓN EN LA EDUCACIÓN SUPERIOR \\ EN PAÍSES DE LATINOAMÉRICA, EUROPA \\ ORIENTAL Y EUROPA OCCIDENTAL (1950-2000)}

\section{Introducción}

El presente artículo investiga la relación que existe entre las condiciones políticas nacionales y las posibilidades de inclusión social en la educación superior. El análisis se realiza a partir de una comparación internacional que pasa revista al desarrollo de la cobertura en este nivel educativo en países de Latinoamérica, Europa Oriental y Europa Occidental, con posterioridad a la Segunda Guerra Mundial.

La pregunta que se busca responder intenta determinar si bajo condiciones políticas de dictadura y democracia se producen patrones específicos de inclusión en la educación terciaria y cómo estos pueden ser explicados desde la perspectiva de las teorías sociológicas.

Para desarrollar el análisis se comparan los patrones evolutivos de la matrícula de educación superior en los países de Europa Occidental, con los de los países de Europa del Este que funcionaron bajo un régimen de socialismo de Estado y países latinoamericanos que sufrieron dictaduras militares.

\section{Análisis neoinstitucionales de la expansión de la educación superior}

Como punto de partida de esta investigación se han escogido los estudios neoinstitucionales acerca de la expansión de la educación superior, ya que la influencia de las condiciones sociales en el desarrollo de este nivel educativo ha sido lo que se ha investigado y comparado internacionalmente, en especial desde esta perspectiva (Meyer, Ramírez, Rubinson, Boli-Bennet, 1979; Ramírez y Meyer, 
1980; Ramírez y Riddle, 1991; Chabbott y Ramírez, 2000; Schofer y Meyer, 2005; Meyer, Ramírez, Frank, Schofer, 2007).

La aproximación neoinstitucional conocida como world polity approach (enfoque de la polis mundial ${ }^{4}$ ) analiza la expansión de la educación superior no sobre la base de casos de estudio nacionales, sino en el marco de estudios longitudinales comparativos internacionales (cross-national longitudinal studies), con el fin de poder arribar a proposiciones generales acerca de las condiciones sociales del desarrollo de la educación terciaria. Esto significa que los estudios empíricos trabajan con datos de series de tiempo, aplicados a muchos países y por largos periodos.

Los autores demuestran que después de la Segunda Guerra Mundial, en todas las regiones del mundo la participación en la educación superior aumentó de manera constante. La inclusión en las instituciones educativas de este nivel asume en consecuencia y de manera universal la forma de una expansión sistemática. Además de ello, se parte de la premisa de que dicha expansión, en lo esencial, es independiente de las condiciones económicas y políticas nacionales (una presentación y análisis detallado de los procedimientos metodológicos de dichas investigaciones y de sus principales hallazgos se encuentra en Reisz y Stock, 2007).

A partir de estos hallazgos las investigaciones neoinstitucionales extraen dos conclusiones: la primera de ellas es que la expansión no tiene un origen nacional, sino que se trata de un fenómeno global; la segunda es que esta sigue pautas culturales que han adquirido validez en todo el mundo. Ramírez y Riddle (1991) lo señalan del siguiente modo en una investigación internacional acerca de la expansión de la educación terciaria: «Nations states seek to adhere to global doctrines of progress and justice by enacting policies that symbolically affirm their legitimacy within a broader world milieu» ${ }^{5}$. Las doctrinas de progreso y justicia representan así un mito cultural que los autores

4 Traducción propia.

5 Los estados nacionales procuran adherir a doctrinas globales de progreso y justicia, a través de la aprobación de políticas que afirman simbólicamente su legitimidad al interior de un entorno mundial más amplio. (Traducción propia). 
interpretan como una expresión del racionalismo occidental (Meyer, Boli, Thomas, Ramírez, 1997).

La educación, en consecuencia, es vista como un medio para promover la prosperidad nacional, así como el desarrollo de los individuos. Esta idea se difunde en todo el mundo y de ese modo se vuelve parte de una world polity cultural (polis mundial cultural) ${ }^{6}$, que impulsa la expansión de la educación y de las instituciones de educación superior. «Massification of both basic schooling and higher education in the 20th century -así lo resumen Chabbot y Ramírez (2000: 173) - appear to be attuned to transnational blueprints for promoting development through education ${ }^{7}$. La propagación de esos modelos es propiciada por reparticiones supranacionales, como asociaciones y organizaciones internacionales que representan modelos profesionales de educación formal (la UNESCO y otras dependencias de la ONU, la OCDE, el Banco Mundial, etc. forman parte de este tipo de organismos). En ninguna parte del mundo el desarrollo educativo puede sustraerse de las expectativas normativas que representan estas fuerzas.

La conclusión de esta aproximación de la polis mundial, que señala que las instituciones de educación superior se expanden con independencia de las condiciones políticas -y en ellas queremos concentrarnos- fue criticada principalmente por Maxwell (1987), en particular en lo relacionado con el desarrollo de la educación terciaria en China en el periodo de la Revolución Cultural comunista y en

6 Traducción propia.

7 La masificación tanto de la educación básica como de la educación superior en el siglo XX parece estar en sintonía con modelos transnacionales que promueven el desarrollo a través de la educación. (Traducción propia). 
Brasil en el periodo de las dictaduras militares ${ }^{8}$. Maxwell explicita, en vista de los hallazgos en esos países, el supuesto de que: «States characterized by the centralization of political power are likely to exert control over higher education and to limit university enrollments» (Maxwell, 1987: 383) ${ }^{9}$.

En las páginas que siguen someteremos a prueba este supuesto a partir de datos respecto de la participación en educación superior en Latinoamérica y Europa Oriental y Occidental entre los años 1950 y 2000. La selección de países permite comparar de modo sistemático el desarrollo de la inclusión en educación terciaria bajo condiciones de dictadura y democracia. Mientras los efectos de la expansión de la educación e instituciones de educación superior sobre el desarrollo de la democracia ya han sido investigados empíricamente (Kamens, 1988; Benavot, 1996; Kamens, 2009), faltan aún análisis empíricos que, a la inversa, analicen comparativamente en diversos países las condiciones políticas necesarias para que dicha expansión tenga lugar.

8 "The case of China illustrates some of the relevant considerations. China was not included in the higher education statistics tables of many editions of the UNESO handbooks, or in many of the recent multiple-regression studies of enrolments. If one were to use data points only at the conventional five- or ten year points as the beginning and middle of decades, rather than observations as dictated by theoretical considerations, one could miss the fact that Chinese universities were closed and had no enrolments for the years 1966-1969. The conclusion by Meyer et al. that national political factors had little effect on higher education enrollments during the period since the last world war seems generally untenable in the face of the evidence from several of Asian nations (...). When applied to China, such a conclusion appears farfetched". (Maxwell, 1987: 380). (El caso de China ilustra algunas de las consideraciones relevantes. En muchas de las ediciones de los Anuarios de la UNESCO no se incluyó China en las tablas estadísticas sobre educación superior, ni tampoco en muchos de los recientes estudios de regresión múltiple referidos a matrícula. Si uno solo utilizara como punto de inicio de los datos los convencionales puntos cada cinco o diez años, como el comienzo y fin de una década, en lugar de monitoreos más frecuentes como señalan las consideraciones teóricas, uno podría no enterarse del hecho de que las universidades chinas estuvieron cerradas y no abrieron matrículas en los años 1966 a 1969. La conclusión de Meyer et al. que factores políticos nacionales tienen poco efecto en la matrícula de educación superior en el período que va desde la última guerra mundial aparece como insostenible frente a la evidencia de varias naciones de Asia (...). Cuando dicha conclusión se aplica a China, aparece como descabellada) (Traducción propia).

9 Los Estados que se caracterizan por la centralización del poder político son proclives a ejercer control sobre la educación superior y a limitar la matrícula universitaria. (Traducción propia). 


\section{Inclusión en la educación superior: hallazgos empíricos}

En los siguientes análisis empíricos contrastamos el desarrollo de la educación superior en los países latinoamericanos y de Europa Oriental, con los países industriales de Europa Occidental. En el grupo de países de Europa Oriental se incluyen -aunque en un sentido geográfico estricto esto es algo simplificado- la República Democrática Alemana (en la actualidad los Estados Federales que la conformaron), Polonia, Hungría, Checoslovaquia (actualmente República Checa y Eslovaquia), Rumania, Bulgaria y la Unión Soviética. Por razones de comparabilidad, los Estados que sucedieron a la Unión Soviética no se consideran individualmente. El desarrollo de la educación superior en los países latinoamericanos es analizado en los ejemplos de Argentina, Chile y Brasil. La selección de los países latinoamericanos se orienta, por un lado, por el tamaño de sus sistemas de educación superior y, por otro, porque en dichos países hubo en distintos periodos de su historia dictaduras militares en el poder. Entre los países industriales de Europa Occidental agrupamos Austria, Bélgica, Dinamarca, Finlandia, Francia, Alemania Federal, Irlanda, Italia, Holanda, Noruega, Suecia, Suiza y Gran Bretaña.

La inclusión en la educación superior se mide a partir del número de estudiantes por cada 10.000 habitantes de un país. Estas tasas fueron calculadas para cada año del periodo 1950-2000. Los datos provienen de los Anuarios estadísticos de la UNESCO (1963-2000), así como también se calcularon sobre la base de datos estadísticos de la población y de datos acerca del número de estudiantes en educación superior contenidos tanto en los mencionados anuarios, como en el Statistical Abstract of Latin America. Estas series de tiempo extensas que posibilitan investigar comparativamente la inclusión en la educación superior internacional solo pueden construirse sobre la base del número de estudiantes en relación con el número total de la población. Los datos acerca de la participación en educación superior relacionados con los grupos etarios típicos se encuentran disponibles solo para unos pocos países y solo para los últimos años.

En las investigaciones neoinstitucionales mencionadas, sin embargo, no se aprecian variaciones en las tasas de cobertura, en 
parte porque los intervalos de tiempo entre los levantamientos de información son muy extensos (de hasta 10 años).

Los análisis de las series de tiempo para los países latinoamericanos, europeos occidentales y orientales mencionados arrojan los siguientes resultados:

Gráfico 1: Estudiantes por cada 10.000 habitantes en países de Latinoamérica

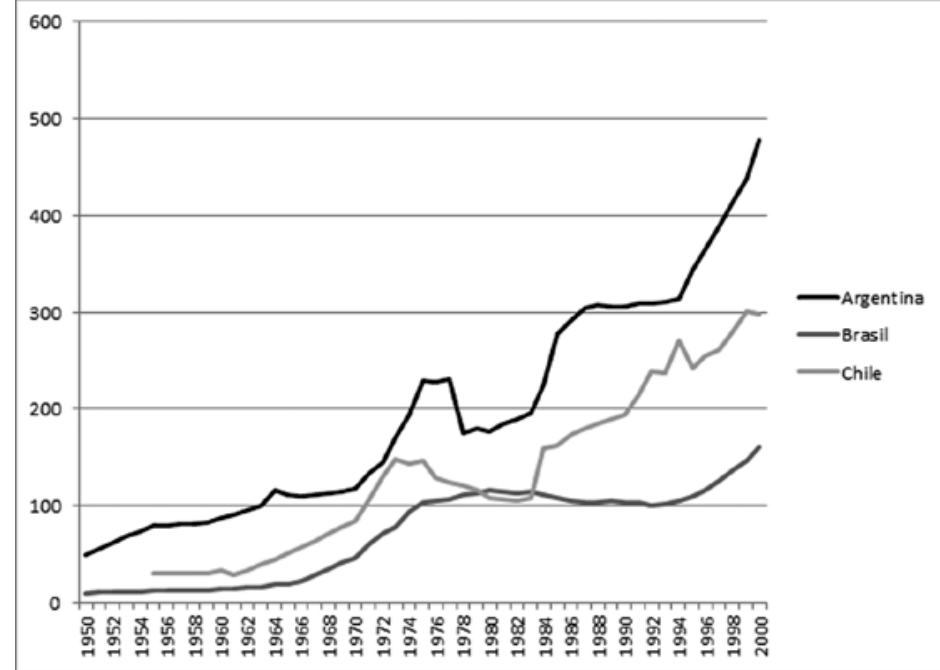

Gráfico 2: Estudiantes por cada 10.000 habitantes en países de Europa Oriental

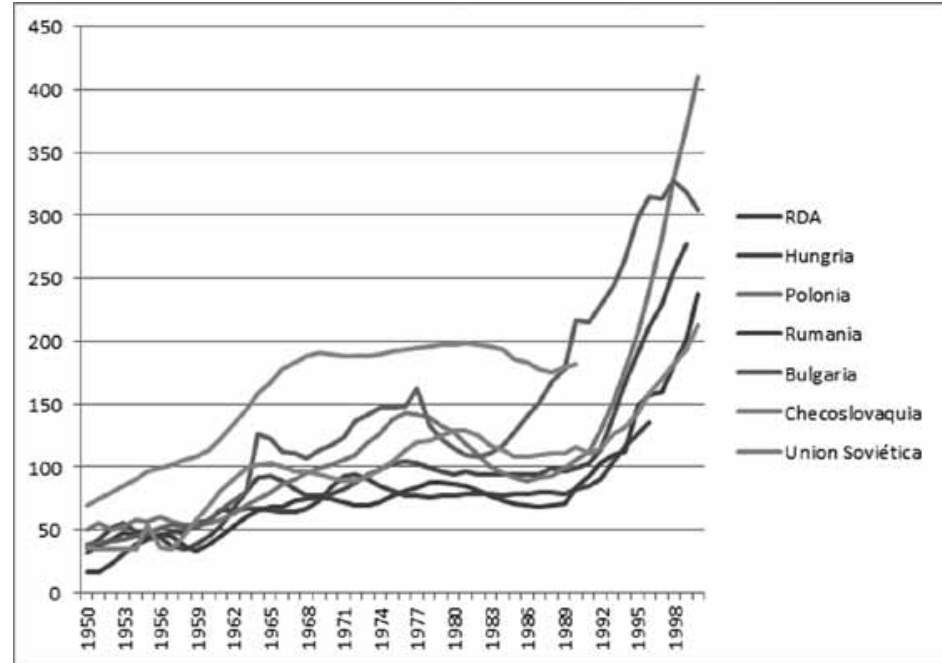


252 ENTRE DEMOCRACIA Y DICTADURA: INCLUSIÓN EN LA EDUCACIÓN SUPERIOR EN PAÍSES DE LATINOAMÉRICA, EUROPA ORIENTAL Y EUROPA OCCIDENTAL (1950-2000) E. Fernández, R. Reisz, M. Stock

Gráfico 3: Estudiantes por cada 10.000 habitantes en países de Europa Occidental

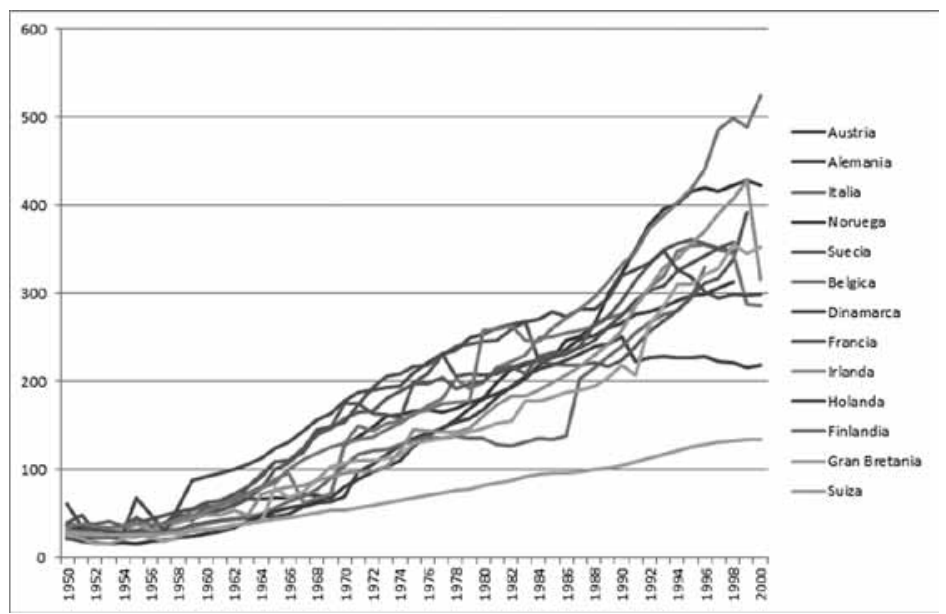

El Gráfico 1 muestra la tasa de participación para cada uno de los países de Europa Oriental; el Gráfico 2, la tasa de participación para los países latinoamericanos; y el Gráfico 3, la tasa de participación para los países industriales desarrollados de Europa Occidental.

La evolución en Europa Occidental muestra en conjunto una expansión constante de la educación superior, la cual encarna el patrón de comportamiento que los análisis neoinstitucionales del enfoque de la polis mundial atribuyen, en general, a la sociedad mundial (Schofer y Meyer, 2005). No obstante, también aquí se puede determinar en algunos pocos países ciertas desviaciones de esta tendencia expansiva continua. Sin embargo, dichas desviaciones no son atribuibles a un retroceso real de la participación en la educación, sino mayormente a los efectos estadísticos producidos por el sistema de clasificación de la 
$\mathrm{UNESCO}^{10}$. Así, en ningún caso las desviaciones estarían relacionadas con cambios estructurales en las relaciones políticas de los países. En el caso de la República Federal Alemana, por ejemplo, a partir de 1991 el número de estudiantes, calculados sobre 10.000 habitantes, se redujo por un breve tiempo, lo cual correspondería a un efecto de la unificación con la República Democrática Alemana. En ese sentido, este resultado no es atribuible a un cambio político en los antiguos estados federales, sino a la repentina baja en la cobertura general, la cual se debe a que la participación estudiantil, como proporción de la población general, en la RDA era mucho más reducida que en la República Federal.

En los países de Europa del Este, en cambio, luego de una fase de expansión hubo una de contracción, sobre todo a partir de la mitad de los años 70. Luego de 1989 en todos los países se produce una nueva expansión de la educación superior, cuyo gradiente se corresponde con el de Europa Occidental. Una alternancia de fases de expansión y contracción también se constata en los países latinoamericanos investigados.

El desarrollo de la educación superior tanto en Europa Oriental como en los países latinoamericanos tiene, entonces, fases de una

10 En el caso de Holanda, de acuerdo con las estadísticas de la UNESCO, se produce a partir de 1993 un retroceso en las tasas de cobertura calculadas por cada 10.000 habitantes, lo que dura hasta 1998. Al sistema de educación superior terciario holandés pertenecen las universidades y las universidades técnicas (HBOs, Hoger Beroepsonderwijs), así como también las escuelas de formación técnica (MBOs) (Statistics Netherlands 2010, Weert y Boezerooy, 2007). En las estadísticas holandesas solo se consideran las universidades y las HBOs como instancias de educación superior. En las estadísticas de la UNESCO también se considera en este nivel una parte de los MBOs. No existe evidencia de que la participación educativa en el ámbito de los HBOs haya retrocedido. Tampoco existen señales de un retroceso en las estadísticas holandesas en lo que respecta a estudiantes de primer año (Weert y Boezerooy, 2007). En cualquier caso, la duración de los estudios evidentemente se ha reducido. A mediados de los años 90 se introdujeron en los Países Bajos los denominados performance grants (subvenciones y préstamos asociados al rendimiento académico) (Weert y Boezerooy, 2007), que recompensaban el acortamiento de los estudios. De igual modo, la introducción de aranceles de estudio, así como su constante alza en el periodo en esta investigación, puede haber contribuido a reducir el tiempo de estudio. La reducción del número total de estudiantes podría ser atribuible a la reducción de los tiempos de estudio. A partir de 1998, es decir, luego de que las «antiguas» cohortes de estudiantes dejaron la universidad, este efecto parece no jugar ningún rol más. Además de ello, a partir de 1999 los criterios para la entrega de performance grants se flexibilizaron. 
masiva contracción en la participación educativa, que se desvía del patrón de expansión constante de la educación superior observado en general en Europa Occidental.

Una comparación de las correlaciones entre las series de tiempo deja en evidencia que las diferencias en los patrones evolutivos efectivamente son sistemáticas. De esta forma, las correlaciones entre las series de tiempo de la tasa de participación estudiantil al interior del grupo de países del oeste (within-group correlations) se sitúan entre 0,888 y 0,991 (sig.=0.01). Al interior del grupo de los países de Europa del Este ellas alcanzan entre 0,872 y 0,983 (sig.=0.01) y entre el grupo de países de América Latina estas corresponden entre 0,923 y 0,982 (sig.=0.01). En cambio, las correlaciones de las series de datos entre los países de los distintos grupos alcanzan (betweengroups correlations) un valor de entre 0,591 y 0,959 (sig.=0.01). La diferencia entre las correlaciones within y between groups es significativa (Mann-Whitney-Wilcoxon; sig. = 0.000). En el caso de Europa del Este además llama la atención que las tasas en todos los países aumentan dramáticamente a partir de 1989.

¿Cómo es posible explicar esta peculiar alternancia entre la expansión y la contracción de la educación superior, que caracteriza el perfil inclusivo de Europa del Este y Latinoamérica?

\section{Expansión y contracción de la educación superior en el contexto de dictadura y democratización}

\subsection{Desarrollo de la educación superior bajo las condiciones de socialismo de Estado y de la democratización posterior a 1989}

En el socialismo de Estado, el desarrollo de la educación terciaria debía seguir las pautas del partido único gobernante: el comunista. Esas pautas no eran sin embargo uniformes. En los hechos, durante el transcurso del periodo estudiado dominaron objetivos contrapuestos para el desarrollo de la educación superior, representados por diversas fracciones de las elites políticas socialistas. Se llegó a conflictos entre los representantes políticos de orientaciones contrapuestas, los que 
bajo la utilización del poder político interrumpieron la expansión de la educación terciaria.

En los años 50 el desarrollo de la educación socialista de Estado debía orientarse hacia el valor de la igualdad. Con ello, debía imponerse algo más que una simple igualdad formal de las posibilidades educativas, puesto que ella ya había conducido en la sociedad capitalista a resultados desiguales en la práctica. Se pretendía con esto eliminar la desigualdad específica en el acceso a la educación superior de las capas sociales. Es así como en prácticamente todos los países se crearon establecimientos educativos especiales, que debían preparar a niños de círculos obreros y campesinos para iniciar un estudio superior. Con este procedimiento se asociaba también un motivo de legitimación política: las antiguas elites debían ser sustituidas por una nueva, una intelligentzia socialista. De las hasta entonces desfavorecidas capas proletarias debía surgir la elite dirigente de la nueva sociedad. Se pensaba que ellas se caracterizarían en igual medida por su lealtad frente a los nuevos dirigentes y por su conocimiento experto. En todos los Estados del entonces bloque oriental, la expansión de la educación superior, hasta entrados los años 60, estuvo bajo el signo de la igualdad material y del recambio de elites.

Posteriormente se institucionalizó en el bloque oriental el concepto modernizador de «nuevo sistema económico del socialismo». La sociedad se imaginaba como una unidad de subsistemas que seguían sus propias reglas, los que con la ayuda de la ciencia se podrían comprender y estructurar de mejor manera que hasta ese momento. La economía, la ciencia y la educación, que antes debían estar bajo el control central, deberían ahora ganar un cierto grado de autonomía. La ciencia debía desplegarse como una fuerza productiva y con ella también aquellas instituciones en las que esta se reproduce y distribuye: las universidades. Ello significó la introducción de nuevas técnicas directivas, así como la puesta en relación de una planificación y dirección fundadas científicamente según los intereses materiales de todos los involucrados en el proceso productivo. De esta forma, el sistema de remuneraciones se vio fuertemente diferenciado y las desigualdades sociales fueron incluidas en el cálculo como incentivos a la productividad. 

EN PAÍSES DE LATINOAMÉRICA, EUROPA ORIENTAL Y EUROPA OCCIDENTAL (1950-2000) E. Fernández, R. Reisz, M. Stock

El socialismo debía ser impulsado con la ayuda de la ciencia y la técnica moderna, las cuales eran vistas como fuerzas productivas que debían desarrollarse en conformidad con supuestas leyes objetivas y la intelligentzia se comprendía a sí misma como su custodio. Bajo la invocación de estas necesidades objetivas ella quería disponer de la organización del trabajo y de los trabajadores. La expansión de las universidades ahora se ponía en relación con las supuestas reglas de la revolución científico-tecnológica y era interpretada a partir de conceptos económico-educativos. La tesis del requerimiento de fuerza laboral de la economía proveyó las ideas centrales que la política educativa asumió. Así, se partía del supuesto de que las leyes de la revolución científico-tecnológica determinaban el desarrollo de la división del trabajo y, con ello, el desarrollo de los requerimientos de calificación laboral. Además, se pensaba que el progreso haría aumentar las necesidades de calificación profesional.

Correspondientemente con esto, se aumentó de forma especial el número de puestos de estudio para las ciencias de la ingeniería. El experto calificado académicamente fue proclamado como la nueva figura central del desarrollo educativo. En Radovan Richta (1969), originario de Checoslovaquia, los expertos del bloque oriental encontraron a su portavoz más prominente. Su libro, titulado Civilization at the crossroads. Social and human implications of the scientific and technological revolution, aparecido en Praga en 1967, reivindicaba la supremacía de la competencia técnica y, al mismo tiempo, hacía frente al antiguo círculo dirigente que vinculaba el socialismo más bien con la idea de igualdad. Su libro se transformó en un componente central de comprometidas discusiones, especialmente en Hungría, Polonia y naturalmente en Checoslovaquia.

En principio el movimiento de reforma en Polonia y en Hungría siguió orientaciones distintas de las tecnocráticas, pero también ahí se impusieron los esfuerzos tecnócratas de reforma en los años 60, al igual que en la Unión Soviética, en la Alemania Democrática y en Checoslovaquia. Konrádt y Szelényi (1978) describieron este proceso detalladamente. Ellos vieron durante los años 70 en todos los Estados socialistas a la intelligentzia en el 
camino hacia su transformación como clase dominante: «A dream of a scientifically engineered society was unfolding -and this was what we felt was meant by the march of intellectuals toward power», así resumió Szelényi (1986-87: 114) dicha tendencia en una retrospectiva ${ }^{11}$.

Sin embargo los esfuerzos de racionalización tecnocrática fracasaron en todos los países socialistas, los que entraron en conflicto con las normas del igualitarismo proletario. Las fracciones políticas que aparecieron como sus defensoras tomaron poco después el poder y pusieron abrupto final a la reforma. Este cambio asumió formas especialmente dramáticas en aquel país en el que la intelligentzia se había atrevido a ir más lejos: en Checoslovaquia, en el año 1968. Cuando pusieron fin a las reformas en Polonia y en Hungría los partidarios del igualitarismo proletario invocaron a los trabajadores. La derrota de los expertos orientados tecnocráticamente y el fin de la expansión de la educación superior fueron de la mano, debido a que las universidades eran principalmente el lugar social en el que estos se formaban y ganaban en autoridad y desde ella derivaban sus demandas de liderazgo.

También en la Alemania Democrática la expansión de la educación terciaria fue detenida. El cambio de la política fue parte del cambio de Gobierno desde la administración Ulbricht hacia la de Honecker, en el año 1971. En la VIII Convención del SED ${ }^{12}$ Margot Honecker ajustó cuentas con la política educativa vigente hasta ese momento: «Algunas formulaciones en nuestra propaganda», así criticó ella, «despiertan a veces la impresión de que nuestra escuela debería preparar a la juventud en primera línea para un estudio en las universidades y en las universidades técnicas». En lugar de ello debería tratarse ahora de que la educación «asuma de mejor manera su responsabilidad en la preparación de nuevas generaciones de trabajadores especializados altamente calificados» (Bericht, 1971).

11 Se fue desplegando un sueño de una sociedad científicamente organizada -y eso fue lo que nosotros sentimos que pretendía la marcha de los intelectuales en pos del poder-. (Traducción propia).

12 Partido Comunista de Alemania Democrática (Sozialistische Einheitspartei Deutschland). 

EN PAÍSES DE LATINOAMÉRICA, EUROPA ORIENTAL Y EUROPA OCCIDENTAL (1950-2000) E. Fernández, R. Reisz, M. Stock

Ese mismo año, en Alemania Democrática fue limitado administrativamente el acceso a los estudios de educación superior. En 1970 el porcentaje de estudiantes universitarios de primer año de las correspondientes cohortes llegaba casi al 19\%. En los siguientes tres años este se redujo a un 12,6\% (Köhler y Stock, 2004). Si bien en el cese de la expansión de la educación superior se habló acerca de las nuevas generaciones de trabajadores especializados, dicho concepto debe ser comprendido mucho más como un concepto de batalla en contra del ascenso político de la intelligentzia. El nuevo sistema económico fue abandonado y reemplazado por una economía política que estaba fuertemente orientada hacia los planteamientos político-sociales y a la nivelación de ingresos.

La seguridad social fue declarada como la primera fuerza impulsora de la economía. La interrupción de la expansión de la educación superior fue otra vez fundamentada con argumentos económico-educativos. Así las llamadas «necesidades objetivas del desarrollo tecnológico» que antes debían legitimar la expansión de las universidades, en este nuevo contexto debían presentar su contracción como «objetivamente necesaria». Ahora se parte de la premisa de que a partir del desarrollo tecnológico no es posible derivar un aumento de la necesidad de graduados de educación superior (Korn, 1988).

En la práctica, las decisiones acerca de la política educativa no tenían relación con las supuestas leyes objetivas del desarrollo tecnológico, sino que eran una expresión de conflictos políticos entre los expertos posicionados tecnocráticamente y aquella fracción de las elites del partido que apelaba a las virtudes revolucionarias del proletariado y que se sentía obligada a cumplir con el modelo de igualdad material. Ambas fracciones coincidían, sin embargo, en que el interés individual en la educación poco les importaba.

En 1989 las barreras que el socialismo de Estado puso a los intereses educativos individuales cayeron con la llegada de la democracia. En todos los antiguos países del bloque oriental se impuso una vertiginosa expansión de la educación superior. Algunos países como la República Checa, Polonia y Eslovaquia levantaron 
todo tipo de restricciones de acceso, mientras que otros como Hungría, los países Bálticos y Rumania definieron las cuotas de acceso a puestos de estudio financiados estatalmente, aunque dieron libertad a las universidades para matricular estudiantes adicionales contra el pago de aranceles de estudio (Haug y Tauch, 2001). Para las universidades privadas, que comenzaron rápidamente a expandirse, no existieron restricciones de acceso definidas estatalmente (Reisz, 2003).

A partir del análisis de lo sucedido en los países de Europa del Este se puede apreciar que la expansión de la educación superior está estrechamente relacionada con la democracia, misma, la que le otorgó autoridad al derecho civil de acceso a la educación y con ello generó la base institucional de la expansión de las universidades. Frente a este contexto, luego de la caída del socialismo de Estado, la evolución expansiva de la educación terciaria de los países de Europa Oriental se aproximó a la de los países de Europa Occidental (ver Gráficos 1 y 3).

En las democracias occidentales, en cambio, la participación en la educación superior con posterioridad a la Segunda Guerra Mundial aumentó más o menos continuamente hasta hoy. A la limitación de la educación individual a través de preceptos políticos estatales y a una reducción de los puestos de estudio se contraponen aquí los derechos civiles, por ejemplo, la libertad de educación y formación, la libertad científica, la libertad profesional, la libertad contractual, solo por mencionar los principios más importantes, los cuales no permiten una limitación de la demanda educativa individual.

\subsection{Desarrollo de la educación superior bajo condiciones de dictadura militar y democratización}

En las líneas que siguen se analiza el desarrollo de la educación superior en Argentina, Brasil y Chile.

Si bien en el periodo que se conoce como la Revolución argentina (1966-1973) hubo una cierta expansión de la educación superior, es recién con el fin de la dictadura militar en 1973 cuando 
esta experimenta un crecimiento más notorio ${ }^{13}$. Incluso en una breve fase intermedia de democratización se promulgó en 1974 la Ley nº 20.564 (bajo el gobierno de Perón), que recurre a las universidades para el desarrollo democrático y económico del país. Durante esta etapa se eliminaron, además, las pruebas de ingreso a la universidad con el fin de aumentar la participación en este nivel educativo (Buchbinder, 2005).

La nueva toma del poder por parte de los militares en 1976 puso un abrupto final a la expansión de la educación superior. Las universidades eran vistas como un espacio de resistencia contra la junta militar y se les despojó de la autonomía (Balán, 1992). En una primera etapa de la dictadura se persiguió masivamente a alumnos y a profesores, a quienes se les atribuía la resistencia política. En una segunda etapa se introdujeron por ley (Ley de Facto $N^{\circ} 22.207$ ) nuevas reglas administrativas, las que abolieron la autonomía de las universidades. En el año 1977 se redujo, también por vía administrativa, el número de puestos de estudio en un 24\% (Rodríguez y Soprano, 2009). Posteriormente las cuotas de participación en estudios superiores retrocedieron dramáticamente. Recién luego del fin de la dictadura en 1983 y con el inicio de la democracia comenzó un repunte de la expansión de la educación superior que dura hasta hoy (Fernández Lamarra, 2003).

En Chile se aprecia claramente una contracción de las universidades a partir de 1973, o sea, inmediatamente después del golpe militar. También aquí la autonomía universitaria fue abolida de facto por los nuevos gobernantes. Los presupuestos estatales fueron fuertemente reducidos y fueron instalados militares como rectores universitarios delegados (McGinn y Toro, 1977), los que estaban facultados para decidir acerca de la provisión de todos los cargos e impusieron drásticas intervenciones: más de 2.000 personas sindicadas como «profesores

13 De acuerdo con las estadísticas de la época, antes del fin de la dictadura la matrícula creció en todas las universidades nacionales, aunque moderadamente. Incluso, en el mismo periodo se crearon 16 nuevas universidades nacionales en el marco del proyecto «Nuevas universidades para un país». Sin embargo, esta etapa está marcada por las políticas dictatoriales, que perseguían alumnos y profesores en un intento por despolitizar las instituciones argentinas, por lo mismo, a juicio de los investigadores, estas no alcanzaron un gran desarrollo (Muscará, 2010: 118-125, T.2.). 
liberales» fueron desvinculadas del trabajo universitario; alrededor de 20.000 estudiantes fueron expulsados de las universidades; sobre todo las especialidades de ciencias sociales fueron cerradas o fuertemente limitadas en su trabajo investigativo y docente (Brunner, 1986; Garretón y Pozo, 1984). Entre 1973 y 1980 no hubo un fundamento jurídico formal para el funcionamiento de las universidades. Bajo el dictado de los rectores militares, los cuerpos colegiados internos fueron abolidos y se instaló un sistema permanente de vigilancia (Brunner, 1986). El Consejo de rectores fue transformado en un gremio de planificación del desarrollo de la educación superior nacional y en un subordinado a la junta de gobierno (McGinn y Toro, 1977).

En un discurso televisivo en 1976, el general Gustavo Leigh resumió las directrices de la política de educación superior nacional en relación con la autonomía de las universidades con las siguientes palabras:

Because autonomy has a close connection with responsibility, it is naturally regulated by the values and objectives of university policy and at the same time the juridical rule of the country. It cannot, therefore, imply either personal or territorial sanctuary for the realization of actions incompatible with a society ruled by law, that would also be denied to the majority of the citizenry. Nor can it signify protection for political activity within the university confines, above all in the form of oral or written propaganda, relative to issues that are commonly understood as part of political debate and commitment. This limitation also applies to teaching that exceeds natural limits of objective information and reasoned discussion of doctrines and points of view (McGinn y Toro, 1977: 887) ${ }^{14}$.

14 Dado que la autonomía tiene una estrecha conexión con la responsabilidad, está naturalmente regulada por los valores y objetivos de la política universitaria y, al mismo tiempo, las normas jurídicas del país. Esta no puede, por lo tanto, transformarse ni en un refugio personal ni territorial para la realización de acciones incompatibles con una sociedad de derecho, lo que será además rechazado por la mayoría de los ciudadanos. Esta tampoco puede significar una protección para realizar actividad política al interior de la universidad, sobre todo en la forma oral o de propaganda escrita, relativa a aspectos que comúnmente son entendidos como parte del debate y compromiso político. Esta limitación también se aplica a la (...) docencia que excede los límites naturales de la información y discusión razonada acerca de doctrinas y puntos de vista. (Traducción propia). 
Los militares insistieron en que las universidades debían transmitir un conocimiento que tuviera una relevancia práctica y profesional directa, en especial en las profesiones técnicas. Concordantemente se ampliaron los programas de estudio de ciencias de la ingeniería (Schiefelbein, 1992), lo que tuvo como consecuencia un aumento del número de estudiantes a partir de 1983, es decir, durante la dictadura militar. Sin embargo, en el periodo 19751980 el número de alumnos universitarios había caído en un 19\% (Echeverría, 1982). Recién luego del fin de la dictadura en el año 1990, la participación en la educación superior aumentó bruscamente, tendencia que se mantiene hasta hoy ${ }^{15}$.

Por su parte en el caso de Brasil las relaciones entre la expansión de la educación superior y el desarrollo político del país son menos evidentes. No obstante, se aprecia que también en este caso la expansión decayó durante la época del régimen militar y continuó recién con la llegada de la democracia.

En este país hubo regímenes militares en el poder desde 1964 hasta 1985. Ante todo, estos gobiernos aspiraron al establecimiento de una política más bien orientada tecnocráticamente (De Souza, 1977), que trataba a las universidades solo en un sentido técnico, es decir, como si fueran recursos para una modernización económica:

At the time of the military takeover in $1964 \ldots$ the pressure for university reform was intense. Committed to promoting national development and international security through a political dictatorship and an economy linked to international markets, the military regime sought to integrate higher education in the modernization process and to diffuse and control campus-based political activity. Beginning in 1966 the government issued a variety of decrees that brought about major changes in the higher education system. The reform process culminated with the

15 No obstante, resulta relevante señalar que durante el mismo periodo el número de universidades aumentó significativamente, pasando de ocho a más de 40 , como resultado de dos procesos: a) la fragmentación de las dos universidades nacionales (Universidad de Chile y Técnica del Estado) y b) la creación de nuevas universidades privadas. 
passage of a wide-ranging reform law in 1968. (...) The reforms also had a decidedly corporatist and authoritarian character, however, in keeping with the interests of a rightwing military government bent on maintaining social order (Plank y Verhine, 2002: 73) ${ }^{16}$.

Como consecuencia de esta política, las universidades se expandieron hasta 1974. La expansión fue asumida principalmente por el sector universitario privado y en gran parte fue forzada por la política de los militares. Luego de ello la participación estudiantil se estancó y retrocedió, lo que persistió también con posterioridad a la sustitución del último régimen militar en el año 1985. En 1988 se aprobó una nueva Constitución, la que aseguró a las universidades una total libertad académica. En 1993, a través de un referendum, se estipuló la reforma estatal de la República de Brasil (Schwartzman, 1992), proceso de democratización que coincide con el inicio de una nueva expansión de las universidades.

Ahora bien, más allá de cómo hayan sido las relaciones causales en cada uno de los países latinoamericanos investigados, se aprecia la existencia de un patrón común de desarrollo de la educación superior: en primera instancia las universidades no se expandieron constantemente $y$, en segundo término, bajo las condiciones de dictadura, culpables de masivas violaciones de los derechos humanos, se experimentaron caídas particularmente dramáticas en la participación en este nivel educativo. En cambio una democratización de las relaciones fue acompañada en todos los países que vivieron una expansión de las universidades.

16 En el tiempo del golpe militar en 1964 (...) la presión para una reforma universitaria fue intensa. Comprometido con la promoción del desarrollo nacional y la seguridad internacional a través de una dictadura política y una economía vinculada a los mercados internacionales, el régimen militar buscó integrar la educación superior en el proceso de modernización y diluir y controlar la actividad política que tenía lugar en los campus. Comenzando en 1966, el Gobierno publicó varios decretos que procuraban realizar importantes cambios en el sistema de educación superior. La reforma culminó con la aprobación de una reforma legal de amplio alcance en 1968. (...) Las reformas, sin embargo, tenían además un decidido carácter corporativista y autoritario, en consonancia con los intereses de un gobierno militar de derecha que procuraba mantener el orden social. (Traducción propia). 
A primera vista puede parecer sorprendente que países que fueron regidos por juntas militares que invocaban valores conservadores o de «derecha», experimentaran una evolución similar respecto del desarrollo de la educación superior que la ocurrida en los países con socialismo de Estado, gobernados por regímenes políticos de «izquierda». Más sorprendente aún si se toma en cuenta que el socialismo se apoyaba en una ideología asumida como «científica». Sin embargo, como lo hemos demostrado más arriba, una observación más cercana hace esta coincidencia comprensible.

En el socialismo la ciencia fue interpretada de manera objetivista. En efecto, en los años 60 la elite partidaria dominante buscaba desarrollar una intelligentzia tecnocráticamente orientada, la que debía poner en práctica las «leyes objetivas del desarrollo de la sociedad». A todo ello le puso fin por razones de poder político aquella fracción que se erigía como representante de los intereses de los trabajadores. Esta fracción no apareció a favor de la libertad de la ciencia o de una formación académica libre, sino que confirmó la comprensión tecnocrática objetivista de la ciencia, en la medida en que la consideró como la legítima base de poder de la intelligentzia y, por lo mismo, limitó el acceso a las universidades e instituciones de educación superior en general.

Recién con la vuelta de la democracia, tanto para el caso de las dictaduras militares como también en lo referente a la caída del socialismo, las universidades se expandieron ininterrumpidamente -como en Europa Occidental- en nombre de la libertad académica y de los derechos civiles, que exigieron un libre acceso a la educación superior. Esta situación sugiere la necesidad de analizar más detalladamente la relación entre la expansión de la educación superior y la democratización. Nosotros emprenderemos este análisis desde dos perspectivas teóricas, por una parte, desde la teoría de la modernización de Talcott Parsons y, por otra, desde la perspectiva de la teoría de la diferenciación funcional. 


\section{Expansión de la educación superior y democratización en la perspectiva de las teorías sociológicas}

\subsection{Teoría de la modernización de Talcott Parsons}

La perspectiva de la democratización se inicia, en primer lugar, con Talcott Parsons, ya que precisamente la relación entre la democratización y la expansión de la educación superior en el periodo posterior a la Segunda Guerra Mundial es señalada por Parsons como punto central de su teoría de la modernización (Parsons, 1971a; Parsons y Platt, 1973). Para Parsons el desarrollo social de la modernidad está determinado por tres revoluciones: inicialmente por la Revolución Industrial; seguida por una revolución democrática; y luego, en el periodo posterior a la Segunda Guerra Mundial, por la revolución educativa.

De acuerdo con Parsons, socioestructuralmente el núcleo de la Revolución Industrial corresponde a la diferenciación de roles laborales y su autonomización de las economías domésticas familiares, así como de la organización empresarial de la producción.

Para el caso de la revolución democrática esta contiene la institucionalización de la «ciudadanía» (citizenship) y los derechos ciudadanos políticos, civiles y sociales con ella asociados. Ambas revoluciones (la industrial y la democrática) tienen para Parsons su punto de partida en el patrón normativo del individualismo institucionalizado, el que encuentra sus raíces en el protestantismo ascético.

Por último, la revolución educativa, que es determinante en la fase actual de la modernidad, se debe a la eficacia de los derechos ciudadanos mencionados. Parsons considera entonces a la revolución educativa no en un sentido económico y educativo o como un reflejo de los requerimientos de calificación laboral resultantes del desarrollo tecnológico y organizacional en el marco de la Revolución Industrial, sino que comprende progresivamente el sector primario, luego el secundario y también el terciario. Desde esta perspectiva, la 
educación superior se transforma así de una educación de elite a una de educación de masas:

It is likely -de esta manera resume Parsons sus análisis acerca de la relación entre el desarrollo de la política, el derecho y la educación superior- that societies which have developed democratic governmental systems have also been relatively hospitable to the development of ... higher education... There also seems to be an important relation between these complexes and the development of legal systems (Parsons, 1971b: 243) ${ }^{17}$.

Para Parsons la estrecha relación entre el desarrollo de las instituciones de educación superior y la democratización resulta de una homología entre la libertad académica y los derechos civiles. En The american university, el autor señala lo siguiente respecto del tema:

The modern university has an affinity with liberal democracy with its pluralization of legitimate political affiliations. (...) rights of the citizen in liberal democratic societies are closely connected with academic freedom, which is the right to conduct cognitive exploration and communication with minimal preimposed constraints (Parsons y Platt, 1973: 293) ${ }^{18}$.

Según Parsons ello está asegurado a través de reglamentos asociados a la validación de la legitimidad de formulaciones argumentales, las que bajo condiciones liberales el autor ve ancladas en los ámbitos académicos, jurídicos y políticos en igual medida, refiriéndose a una «congruencia estructural» (Parsons, 1968: 195).

Ante este transfondo teórico el desarrollo de la educación superior en América Latina y en Europa del Este luego de la superación

17 Es probable que las sociedades que han desarrollado sistemas de gobierno democráticos han sido también relativamente favorables al desarrollo de la educación superior (...). Parece entonces que también existe una importante relación entre dichos complejos y el desarrollo de sistemas legales. (Traducción propia).

18 La universidad moderna tiene una afinidad con la democracia liberal, con su pluralización de afiliaciones políticas legítimas (...); los derechos de los ciudadanos en las sociedades democráticas liberales están estrechamente conectados con la libertad académica, que es el derecho a conducir la exploración y comunicación cognitiva con un mínimo de limitaciones preimpuestas. (Traducción propia). 
de los regímenes dictatoriales después de 1989, aparece como un momento de recuperación de una modernización conducida normativamente. Tampoco estas regiones del mundo podían sustraerse de la presión del patrón normativo occidental universal: el fin de las dictaduras es una expresión de ello. Luego de su término en los distintos países, los derechos civiles ganan en importancia y sobre esta base institucional se expanden también las instituciones de educación superior.

La teoría de Parsons se ubica en sus conceptos fundamentales en la cercanía de la aproximación neoinstitucionalista del análisis de la polis mundial, ya que ambos argumentan a partir de una figura normativa descendente (top-down): en el caso del neoinstitucionalismo este asocia la expansión de la educación superior causalmente con la autoridad normativa de un modelo mundial (world model) de desarrollo de la educación superior (Reisz y Stock, 2007); mientras que Parsons lo asocia con el patrón normativo universal del individualismo institucionalizado (Stock, 2005).

Efectivamente, tanto en los países de Europa del Este como en los de América Latina, la aproximación experimentada hacia el curso seguido por la inclusión educativa de los países occidentales denota el carácter global del desarrollo de la educación, el que las investigaciones neoinstitucionalistas destacan. Sin embargo, al mismo tiempo su desarrollo en esos países hace visibles los límites de la aproximación neoinstitucional.

Las condiciones socioestructurales que llevaron a la interrupción de la expansión educativa en los países del bloque oriental y bajo las dictaduras militares latinoamericanas quedan fuera de su campo visual. Tampoco la teoría de Parsons contribuye en esta dirección. Ambas teorías ven en este quiebre solo la desviación de una tendencia modernizadora universal que sigue normas típicamente occidentales. La desviación en sí misma no puede ser explicada estructuralmente a partir de estas teorías: para aproximarnos a dicha explicación, recurriremos a una interpretación a partir de los conceptos de la teoría de la diferenciación funcional. 


\subsection{Teoría de la diferenciación funcional y sociedad mundial}

Como hemos dicho anteriormente, tanto en el bloque oriental como en las dictaduras militares de Latinoamérica, la expansión de la educación superior fue anulada o significativamente reducida a través de las decisiones políticas que tomaron sus respectivos gobiernos. Mientras la autonomía funcional de la ciencia y la educación se corresponde con un reconocimiento de la demanda individual por educación, en el caso del socialismo se intentó alinearla con las supuestas «exigencias del desarrollo social» en tanto que bajo las dictaduras militares se le limitó simplemente por las definiciones de poder político, debido a que las universidades eran sindicadas como espacios de resistencia.

Esta preeminencia de lo político fue abolida tanto con la caída del socialismo de Estado como con el fin de las dictaduras militares. Los mecanismos de inclusión en el sistema de educación superior fueron ahora sustraídos a la intervención política directa y a los intereses educativos individuales para que pudieran articularse. Estos cambios socioestructurales son invisibilizados tanto en la perspectiva de la teoría neoinstitucional (que analiza la sociedad mundial desde el punto de vista de la difusión e institucionalización de los estándares normativos y cognitivos), como también en la teoría argumentativamente normativa de la modernización de Parsons.

Si la transformación esbozada se analiza desde una perspectiva de la teoría de la diferenciación (Luhmann, 1997a), esta se presenta como el componente de una emancipación del sistema educativo de la política, que libera la lógica funcional de este ámbito. Socialismo de Estado y dictaduras militares deberían, entonces, ser analizadas en primer lugar desde el punto de vista de la desdiferenciación funcional (entre otros, Luhmann, 1997a; Wilke, 2000).

Una perspectiva como esta expresa también que las discusiones presentadas durante el tiempo del socialismo de Estado, por un lado, estaban directamente unidas a la pregunta por la relación entre los aspectos sistémicos específicos de la lógica de funcionamiento de la economía o de la ciencia y, por otro, por las programaciones de 
intervención política general del sistema ${ }^{19}$. Las elites posicionadas tecnocráticamente representaban la lógica funcional de los distintos ámbitos sociales ${ }^{20}$, en tanto que la elite dominante representaba más bien los esfuerzos de su programación política general.

En la perspectiva teórica de la diferenciación, la transformación postsocialista y la democratización luego del fin de las dictaduras militares en los países de América Latina se ubican en el centro como transformaciones que ponen a disposición de estas regiones la dinámica de una sociedad mundial diferenciada funcionalmente $e^{21}$. No obstante, esto no significa que no persistan las diferencias regionales (Luhmann, 2005).

Desde esta perspectiva es posible poner en relación el perfil inclusivo que hemos podido comprobar para Europa Occidental y que se caracteriza por una relativa gran continuidad con una estructura social funcionalmente diferenciada. Se trata en este caso de una inclusión de las personas en el sistema educativo, que está dirigida a ellas en tanto individuos ${ }^{22}$. Los altos y bajos del perfil inclusivo de los países del bloque oriental hasta 1989 y de los países latinoamericanos investigados, se corresponden en cambio con hechos estructurales, bajo los que el desarrollo de los sistemas educativos y de educación superior debían seguir determinaciones y programaciones políticas. Luego de la caída del socialismo y del fin de las dictaduras militares, estos perfiles inclusivos se asimilaron a aquel que se había impuesto en Occidente bajo condiciones de diferenciación funcional.

19 En las investigaciones acerca del socialismo de Estado y su transformación, esta relación es extensamente discutida como un "conflicto entre lógicas políticas y objetivas» (Pollack, 2002: 47).

20 Un intento por vincular el tema de las elites con las teorías de la diferenciación funcional se encuentra en Nassehi (2004).

21 La diferenciación funcional no conduce a una simple autonomía del sistema educativo o científico de la política y de otros sistemas funcionales, sino también a dependencias específicas, lo que muestra la observación acerca de la institucionalización de un derecho civil a la educación. En los conceptos de la teoría de la diferenciación funcional esto sería un mecanismo de acoplamiento estructural entre los sistemas educativo y jurídico (Luhmann, 1997a).

22 Para el análisis de una inclusión individualizadora en los sistemas funcionales, ver Nassehi (2003). 
Si se siguen los argumentos de la teoría de sistemas, entonces, los sistemas funcionales como la economía, la ciencia, el deporte, etc., se expanden sobre sus propios y respectivos fundamentos y despliegan una tendencia a la formación de operaciones y estructuras a nivel global (Luhmann, 1997ab y 2005). La expansión es una consecuencia de la autonomización y autoestabilización de la respectiva lógica funcional con la que se relaciona una creciente inclusión de las personas y un aumento del número de población.

La perspectiva de la diferenciación de la teoría de sistemas no observa a la sociedad mundial como un complejo de sociedades regionales, que se integra normativamente mediante la orientación hacia modelos globales. Del mismo modo, la unidad de la sociedad mundial no está determinada culturalmente en tanto parte de una polis mundial, sino que es vista como una unidad operativa. Cada comunicación sistémica específica implica, conforme con ello, una sociedad mundial, ya que ella usa medios simbólicos de intercambio tales como la verdad científica, el dinero y el arte, entre otros, los que no se dejan fijar en fronteras territoriales ${ }^{23}$. Al contrario de la aproximación neoinstitucional y de la teoría de Parsons, la «cultura» no es vista como una causa última del desarrollo de la sociedad global.

\section{Conclusiones}

En relación con las instituciones de educación superior, la presente investigación ofrece un primer punto de referencia para responder a la pregunta por la evolución que ha seguido la inclusión de las personas en el sistema funcional de educación y formación en una comparación entre regiones, en las que la diferenciación funcional en el periodo posterior a la Segunda Guerra Mundial ha tenido impulsos de distinta magnitud.

El presente estudio comparativo investigó el curso seguido por los procesos de inclusión en las instituciones de educación superior, en dictadura y en democracia. En condiciones democráticas, las universidades se expanden, mientras que bajo dictadura dicha

23 Esto puede ser aquí solo enunciado. Mayor detalle en Luhamnn, 2005. 
expansión se detiene o incluso se aprecian fenómenos de contracción de la matrícula.

Esta situación, como se ha podido mostrar, se puede explicar a partir de conceptos de una teoría de la diferenciación funcional. La contracción de las instituciones de educación superior, de acuerdo con esta perspectiva teórica, surge de una estructura social en la que la lógica funcional del sistema educativo es constreñida por la vía de una programación política de gran alcance.

Los distintos cursos que ha seguido la inclusión en la educación superior en el sistema funcional de la educación comparando regiones y fases históricas pueden así ser comprendidos como una expresión de relaciones sociales, en las que la diferenciación funcional en el periodo posterior a la segunda Guerra Mundial tuvo distintas intensidades y apoyo.

\section{Referencias}

Balán, J., (1992) Argentina. En The Encyclopedia of Higher Education (v.1). Edición a cargo de Burton, C. y Guy, N. Oxford / New York: Pergamon Press, pp. 19-29.

Benavot, A., (1996) «Education and Political Democratization: Cross-National and Longitudinal Findings» en: Comparative Education Review, número 40, 4, pp. 377-403.

Bericht des ZK der SED an den VII. Parteitag, 1971. Berlin: Dietz.

Brunner, J., (1986) Informe Sobre Educación Superior en Chile. Flacso, Santiago.

Buchbinder, P., (2005) Historia de las universidades argentinas. Buenos Aires, Editorial Sudamericana.

Chabbott, C. y F. Ramírez, (2000) Development and Education. En Maureen T. Hallinan. (Eds.) Handbook of the Sociology of Education. New York, Kluwer.

De Souza, H., (1977) Brazil. En The International Encyclopedia of Higher Education (v.2). Edición a cargo de Knowels, A. San Francisco / London: Jossey-Bass, pp. 669-676.

Echeverría, R., (1982) Evolución de la matrícula en Chile: 1935-1981. Santiago, PIIE. 
272 ENTRE DEMOCRACIA Y DICTADURA: INCLUSIÓN EN LA EDUCACIÓN SUPERIOR EN PAÍSES DE LATINOAMÉRICA, EUROPA ORIENTAL Y EUROPA OCCIDENTAL (1950-2000) -

E. Fernández, R. Reisz, M. Stock

Fernández Lamarra, N., (2003) Argentina: Equity and Social Inclusion in Higher Education. En Iesalc reports. Bulletin N. 180. International Institute of UNESCO for Higher Education in Latin America and the Caribe, Caracas.

Garretón, M. y H. Pozo, (1984) Las universidades chilenas y los derechos humanos. Santiago, Flacso.

Haug, G. y C. Tauch, (2001) «Trends in Learning Structures in Higher Education (II)». Follow-up Report Prepared for the Salamaca and Prague Conferences of March/May.

Kamens, D., (1988) «Education and Democracy: A Comparative Institutional Analysis» en Sociology of Education, número 61. Abril, pp. 114-127.

Kamens, D., (2009) «The Expanding Polity: Theorizing the Links between Expanded Higher Education and the New Politics of the Post-1970s» en American Journal of Education, número 116. Noviembre, pp. 99-124.

Köhler, H. y M. Stock, (2004) Bildung nach Plan? Bildungs-und Beschäftigungssystem in der DDR 1949 bis 1989. Opladen, Leske und Budrich.

Konrádt, G. y I. Szelényi, (1978) Die Intelligenz auf dem Weg zur Klassenmacht. Frankfurt a.M., Suhrkamp.

Korn, K., (Hrsg.) (1988) «Ökonomie und Planung des Bildungssystems in der intensiv erweiterten Reproduktion der Volkswirtschaft. Protokoll der II». Internationalen Konferenz von Bildungsökonomen sozialistischer Länder, 1988, Berlin.

Luhmann, N., (1997a) Die Gesellschaft der Gesellschaft. Frankfurt a.M., Suhrkamp.

Luhmann, N., (1997b) «Globalization or World Society: How to Conceive of Modern Society? » en International Review of Sociology, número 7, pp. 67-79.

Luhmann, N., (2005) Einführung in die Theorie der Gesellschaft. Heidelberg, Carl-Auer.

Maxwell, W., (1987) «The Expansion of Higher Education Enrollments in Agrarian and Developing Areas of the Third World» en Smart J. (ed.). Higher Education. Handbook of Theory and Research. Nueva York, Agathon Press.

McGinn, N. y E. Toro, (1977) Chile. En The International Encyclopedia of Higher Education (v.2). Edición a cargo de Knowels, A. San Francisco / London: Jossey-Bass, pp. 880-887.

Meyer, J.; Ramírez, F; Rubinson, R. y J. Boli-Bennet, (1979) The World Educational Revolution 1950-70. En Meyer, J. y /Hannan, M. (Hrsg.) 
National Development and the World System. Education, Economic, and Political Change, 1950-1970. Chicago: The University of Chicago Press, pp. 37-55.

Meyer, J.; Boli, J.; Thomas, G. y F. Ramírez, (1997) «World Society and the Nation-State» en American Journal of Sociology, número 103, Julio, pp. 144-181.

Meyer, J.; Ramírez, F.; Frank, D. y E. Schofer, (2007) «Higher Education as an Institution» en Gumport, P. (ed.), Sociology of Higher Education. Baltimore, Johns Hopkins University Press.

Muscará, F., (2010) Educación Superior. Diagnóstico y Propuestas. Cátedra de Política Educativa. Universidad Nacional de Cuyo - Mendoza, Argentina.

Nassehi, A., (2003) Geschlossenheit und Offenheit. Frankfurt a.M., Suhrkamp.

Nassehi, A., (2004) Eliten als Differenzierungsparasiten. Skizze eines Forschungsprogramms en Hitzler, R., Hornbostel, S. y Mohr, C. Elitenmacht (Eds.). Wiesbaden: VS-Verlag, pp. 25-41.

Parsons, T., (1968) «The academic system: a sociologist's view» en The Public Interest, número 13 (special issue), pp. 173-197.

Parsons, T., (1971a) The System of Modern Societies. Englewood Cliffs, Prentice Hall.

Parsons, T., (1971b) «Higher Education as a Theoretical Focus» en Turk, H. y Simpson, R. (eds.), Institutions and Social Change. Indianapolis, Bobbs-Merrill.

Parsons, T. y G. Platt, (1973) The American University. Cambridge, Harvard University Press.

Plank, D. y R. Verhine, (2002) «Flight from Freedom: Resistence to Institutional Autonomy in Brazil's Federal Universities» en Chapman, D. y Austin, A. (eds.), Higher Education in the Developing World. Changing Contexts and Institutional Responses. Boston, Boston College.

Pollack, D. (2002) «War die DDR-Gesellschaft modern? Eine Gesellschaft im Konflikt zwischen politischer Homogenisierung und funktionaler Differenzierung» en SFB 580 Mitteilungen. Gesellschaftliche Entwicklungen nach dem Systemumbruch. Diskontinuität, Tradition und Strukturbildung. Heft 3, September 1995, pp. 45-48.

Ramírez, F. y J. Meyer, (1980) «Comparative Education: The Social Construction of the Modern World System» en Annual Review of Sociology, Volumen 6, pp. 369-399. 
274 ENTRE DEMOCRACIA Y DICTADURA: INCLUSIÓN EN LA EDUCACIÓN SUPERIOR EN PAÍSES DE LATINOAMÉRICA, EUROPA ORIENTAL Y EUROPA OCCIDENTAL (1950-2000) -

E. Fernández, R. Reisz, M. Stock

Ramírez, F. y P. Riddle, (1991) «The Expansion of Higher Education» en Philip G. Altbach (ed.), International Higher Education. An Encyclopedia. New York, Garland Publishing.

Reisz, R., (2003) Public policy for private higher education in Central and Eastern Europe. Conceptual clarifications, statistical evidence, open questions. Wittenberg, Germany, Institut fur Hochschulforschung an der MartinLuther-Universität Halle Wittenberg.

Reisz, R. y M. Stock, (2007) «Theorie der Weltgesellschaft und statistische Modelle im soziologischen Neoinstitutionalismus» en Zeitschrift für Soziologie, número 36, Abril, pp. 82-99.

Richta, R., (1969) Civilization at the Crossroads: Social and Human Implications of the Scientific and Technological Revolution. White Plains, N.Y., International Arts and Sciences.

Rodríguez, L. y G. Soprano, (2009) «La política universitaria de la dictadura militar en la Argentina: proyectos de reestructuración del sistema de educación superior (1976-1983)» en Nuevo Mundo Mundos Nuevo. [En línea]. Universidad de Chile, disponible en: http://nuevomundo.revues. org/index 44042.html [Accesado en febrero del 2010].

Schiefelbein, E., (1992) Chile. En The Encyclopedia of Higher Education (v.1). Edición a cargo de Clark, B. y Neave, G.Oxford/New York: Pergamon Press, pp. 130-137.

Schofer, E. y J. Meyer, (2005) «The World-Wide Expansion of Higher Education in the Twentieth Century» en American Sociological Review, número 70, Diciembre, pp. 898-920.

Schwartzmann, S., (1992) Brazil. En The Encyclopedia of Higher Education (v.1). Edicción a cargo de Clark, B. y Neave. Oxford/New York: Pergamon Press, pp. 82-92.

Statistics Netherlands (2010) «Daten und Definitionen zum Bildungssystem». Statline Centraal Bureau voor de Statistiek [En línea]. Netherlands, disponible en: http://statline.cbs.nl/StatWeb/selection/?VW=T\&DM $=S L E N \& P A=03753 E N G \& D 1=a \& D 2=1-2,16-17 \& D 3=0-2 \& D 4=0 \&$ $\mathrm{D} 5=10,1517 \& \mathrm{LA}=\mathrm{EN} \& H D R=\mathrm{G} 3, \mathrm{~T}, \mathrm{G} 4 \& \mathrm{STB}=\mathrm{G} 2$, G1 [Accesado en marzo del 2010].

Stock, M., (2005) «Hochschule, Professionen und Modernisierung. Zu den professionssoziologischen Analysen Talcott Parsons» en Die Hochschule. Journal für Wissenschaft und Bildung, número 14, (1), pp. 72-91.

Szelényi, I., (1986/1987) «The Prospects and Limits of the East European New Class Project: An Auto-critical Reflection on "The Intellectuals on the 
Road to Class Power"» en Politics and Society, Volumen 15, Número 2, pp. 104-144.

Weert, E. y P. Boezerooy, (2007) Higher education in the Netherlands. Country report, September 2007, CHEPS - International higher education monitor. Netherlands, University of Twente.

Willke, H., (2000) Systemtheorie I: Grundlagen. Stuttgart, Lucius \& Lucius.

Recibido: 5/11/2012

Aceptado: 30/05/2013 